\title{
PROBLEMAS Y RETOS DE LA INVESTIGACION EN EL SIGLO XXI (EL CASO DE LA RIEMS Y LA CONFORMACIÓN DE LA ANTROPOÉTICA)
}

\author{
Víctor Manuel Alvarado Hernández* \\ Martin Manjarrez BetancourT**
}

Recebido em: 21 de dez. 2009 Aprovado em: 9 abr. 2010

\begin{abstract}
* Doutor. en Pedagogia en UNAM. Professor definitivo en Maestría en Pedagogia y Maestría en Derecho, UNAM. E-mail: vicmaah@yahoo.com

** Maestro en Derecho por la Universidad Nacional Autónoma de México (UNAM). Cuenta con el curso de preparación de aspirantes a cargo de Juez por el Instituto de Estudios Judiciales del Tribunal Superior de Justicia. Con la Especialización en Derecho Familiar. Realizó estudios de Licenciatura en Derecho en la Universidad Nacional Autónoma de México (UNAM). E-mail: inedej@hotmail.com
\end{abstract}

Resumen: La investigación en el siglo XXI enfrenta un sin número de retos y problemas, en esta centuria en que vivimos, el reto no sólo de la investigación si no de la educación, es que debe conciliarse la materia y el espíritu, la naturaleza y la cultura, la ciencia y las humanidades, propiciando un reencuentro entre la tecnología y el humanismo, integrando la memoria y el proyecto. Por ello creemos que el nuevo paradigma de la investigación y educación es la conformación de la antropoética. En este documento abordamos la problemática que representa la Reforma Integral de la Educación Media Superior (RIEMS), no sólo para la investigación, sino también para el proceso de conformación de la antropoética. Para ello se abordan sus términos conceptuales, como antropoética, competencias transversales y las nociones de conformación, complejidad. Hemos seleccionado el escenario de la educación media superior, específicamente el bachillerato, porque es en este nivel de la educación, donde se generan diversas modalidades educativas que derivan en prácticas neoliberalistas de capacitación e instrucción, tal es el caso de la RIEMS, lo que inhibe el proceso de formación humana y por ende su posibilidad de formación antropoética. Esta ultima como una opción clave para la educación de las futuras generaciones.

Palabras clave: Antropoética. Investigación. RIEMS. Competencias. Transversales. Complejidad. Educación media superior.

\author{
PROBLEMS AND CHALLENGES OF RESEARCH IN THE XXI CENTURY \\ (THE CASE OF THE Riems AND CONFORMATION OF THE ANTHROPOETICS)
}

Abstract. Research in the XXI century faces a myriad of challenges and problems, in this century in which we live, the challenge not only of research but also of education, is that they must reconcile matter and spirit, nature and culture, science and humanities, leading to an reencounter between technology and humanism, integrating memory and project. We therefore believe that the new paradigm of research and education is the conformation to anthropoetics. In this paper we address the problem posed by the Comprehensive Reform for Middle and Higher Education (Riems), not only for research but also for the process of shaping the anthropoetics. This will address conceptual terms, as anthropoetics, generic skills and concepts of structure, complexity. We have chosen the scenario of higher education, undergratuate specifically, because it is at this level of education, that are generated various types of education that result in practical neoliberal training and instruction, such is the case of Riems, which inhibits human education processes and thus their ability of anthropoetic training. This latter as a key option for the education of future generations.

Keywords: Anthropoetics. Research. RIEMS. Competencies. Transverse. Complexity. Higher Education. 


\section{INTRODUCCIÓN}

La investigación al igual que la educación se han alejado del postulado de formar al hombre como un ser humano consciente de su entorno y su contexto, de ser un individuo perteneciente a una sociedad y a una especie, en este sentido se han provocado serios y graves problemas como el daño ecológico, social y moral; sobrepoblación; la brecha cada vez más amplia y nítida de la desigualdad; el materialismo creciente. Así con el erróneo postulado reduccionista de que conocer es la forma máxima para dominar, la asociación equivoca de ideas entre conocimiento y utilidad (MORÍN, 2000), han llevado a las autoridades educativas de diversos países a la denominada "Economía del conocimiento", para ello han adoptado políticas educativas como la RIEMS, en el caso de México, que tienden a establecer el modelo educativo por competencias, destinado no a la conformación antropoética de los jóvenes estudiantes, a la formación de seres humanos integrales, si no a la capacitación de mano de obra técnicamente calificada para la producción.

Todo ello ha contribuido a que pese sobre el hombre la amenaza de su autodestrucción. Todos esos poderes extraordinarios que tiene la humanidad de construirse a sí misma y su destino, la han llevado a caer en un desequilibrio, el hombre se incendia así mismo.

Ante ese oscuro y tenebroso panorama, se ha tratado de aportar con el presente trabajo una forma de permitir la realización del hombre con la investigación humanista, cuyo aporte sea la conformación de la Antropoética. Ya que es precisamente en este rubro de la educación y la investigación humanística donde consideramos se plasma la esperanza de salvaguardar a la humanidad.

Para ello se exhorta a la reflexión de la problemática que representa la RIEMS para la educación y de sus procesos de investigación en general y específicamente para la investigación en las humanidades, así como para la conformación de la antropoética, por lo que se establece un marco conceptual donde se aborda categorías importantes como competencias, transversalidad, complejidad, comprensión y antropoética, mismas que servirán para desarrollar y explicar cómo en la medida que la educación media superior se enfoque a investigar y trabajar para la humanización de la humanidad, nos acercaremos a efectuar la doble conversión de la educación; recibir educación para obedecer a su naturaleza de vida, y para el día de mañana ser los investigadores científicos, los orientadores y guías de la vida, lograr el reconocimiento de la unidad planetaria en la diversidad individual, la enseñanza del respeto al otro, a la vez, tanto la diferencia como la identidad consigo mismo, la enseñanza y desarrollo de la ética, en su triple aspecto, de la solidaridad, de la comprensión y de la ética del género humano. (MORÍN, 2001) 
Es así que desarrollando todos estos aspectos en sus diversas fases se trato de consensuar que para la investigación uno de sus retos es la conformación antropoética de los jóvenes estudiantes de la educación media superior, no siendo posible sin la enseñanza de las humanidades, disciplinas que se ven amenazadas por la RIEMS, sin reparar que son sumamente esenciales, por que conllevan a la esperanza de lograr la humanización de la humanidad, es decir, la esperanza de preservar y salvaguardar a la humanidad, a través de la educación y la investigación que les permita realizarse.

\section{PLANTEAMIENTO DEL PROBLEMA}

El siglo XXI ha lanzado serios retos, para todas las áreas del conocimiento, pero en especial para la investigación, la cual deberá contemplar la conformación antropoética para la formación de seres humanos y no sólo personas competentes como lo pretende la RIEMS, representando está última una verdadera problemática para la investigación, ya que con la RIEMS se ha desplazado a la filosofía y a otras disciplinas de las humanidades, como no básicas, otorgándoles el carácter de "transversales", poniéndose en grave riesgo, la investigación humanística, por lo que el problema no es la reforma educativa, que en ese tópico todos los tratadistas están de acuerdo y proclaman su urgencia, si no que en aras de una apremiante necesidad de una reforma educativa se ha excluido a la filosofía y a otras disciplinas humanísticas de las disciplinas básicas del Sistema Nacional de Bachillerato (SNB) lo que repercute en la investigación humanística tal y como lo señalan cinco destacados tratadistas filósofos (Luis Villoro, Ramón Xirau, Alejandro Rossi, Adolfo Sánchez Vásquez y José Luis Balcárcel (PONCE, 2009), que la Filosofía es la base de todas las ciencias y de todo conocimiento, agrega Ramón Xirau...sin ella no hay posibilidad de conocer nada de lo demás. Si hoy a las jóvenes generaciones de alumnos, quienes serán los investigadores del mañana, no se les enseña disciplinas humanísticas tan esenciales como la lógica, la ética, la estética y la filosofía, difícilmente llegarán a serlo, es por ello que fue necesario plantearnos las siguientes interrogantes: $¿ \mathrm{La}$ crisis del humanismo es un problema o un reto de la investigación?, para el caso de resultar positiva la respuesta ¿Cómo puede la investigación enfrentar ese reto?

\section{OBJETIVO}

Analizar algunas problemáticas y retos de la investigación en las humanidades a través de la conformación antropoética a fin de señalar las deficiencias de formación establecidas en la RIEMS. 


\section{HIPÓTESIS}

La investigación en el siglo XXI enfrenta grandes retos y problemáticas por lo que creemos que para su enfrentamiento deberá afrontar una reforma educativa que permita la formación integral humana de los alumnos que en el mañana serán los futuros investigadores, por ello no basta implementar una Reforma Integral de la Educación Media Superior con una visión dogmáticamente reduccionista, si no una reforma pluridisciplinaria que permita el conocimiento complejo para la conformación antropoética de los jóvenes estudiantes.

\section{METODOLOGIA}

En el presente trabajo se parte de los umbrales del Siglo XXI para ver cuáles son los retos y la problemática por afrontar de la investigación, para detectar que uno de sus retos es la deshumanización de los estudiantes, así vislumbramos, que la investigación no sólo aporta, sino forma a los alumnos quienes serán futuros investigadores. En este sentido, la investigación deberá formar investigadores como seres humanos integrales a través de la antropoética, por lo que se aborda el caso de la RIEMS que viene a representar una problemática que pretende sustraer las disciplinas humanísticas de la Educación Media Superior cuestionando la pertinencia de dicha medida para desentrañar sus repercusiones en la formación antropoética de los futuros investigadores.

\section{MÉTODO}

El método más empleado en la presente investigación es el analítico, ello no implica que no se utilicen otros métodos como el histórico, deductivo y sociológico. Sin embargo, para desentrañar lo del pensamiento es necesario utilizar el método analítico para reflexionar sobre lo desarrollado por el tratadista francés Edgar Morin (2005), que nos sirve para demostrar que los modos simplificadores del conocimiento producen más problemas que elucidación, como es el caso de la RIEMS, que lejos de ser una solución para el debate educativo de la investigación y con el cual está pueda afrontar la crisis de la humanidad, ha traído más problemas para la formación e investigación.

\section{DESARROLLO}

\section{La crisis del humanismo y la conformación antropoética como retos de la investigación}

A la problemática propia de la investigación en México (reducción de presupuesto, falta de capital humano, reducción del producto interno bruto y por ende de lo destinado a la investigación, fuga de cerebros, así como una casi 
nula formación de estudiantes nacionales en el extranjero, etc.) A ello se le ha añadido la crisis del humanismo, que como afirma la filósofa Juliana González: "Estamos cerrando la modernidad y al mismo tiempo nos abrimos a un futuro mucho más desconocido. Quizá el futuro nunca ha sido más incógnito como puede ser en nuestro propio tiempo" (ROMERO, 2005, p. 10). Para algunos pensadores y filósofos (como la citada académica), estamos llegando al fin del humanismo, donde el hombre como género deja de ser lo más importante, el centro del universo. De acuerdo a Morin (2000), el hombre no debe verse como un ser jerárquicamente superior, sino como un individuo perteneciente a una sociedad y a una especie para poder convivir en armonía con su comunidad y ecosistema.

De esta idea del hombre jerárquicamente superior surge los siguientes cuestionamientos: ¿La crisis del humanismo es un problema o es un reto para la investigación? ¿Cómo puede la investigación atender esta crisis del humanismo? ¿Qué puede aportar la investigación para generar un neohumanismo?

Creemos que la crisis del humanismo no es propiamente un problema de la investigación en humanidades, sino que es un reto que debe afrontar en virtud de que la investigación es parte del debate pedagógico (LUCINI, 1995), que hoy nos apremia, que se plantea el cómo enfrentar desde el escenario de la educación esa crisis que a todos nos compete y compromete a favorecer y potenciar una sólida educación moral, ecológica, social y humanística de la infancia y la juventud, quienes serán los artífices del futuro, los investigadores del mañana.

En lo que respecta a cómo puede la investigación atender esta crisis del humanismo, creemos que la respuesta es la sugerencia que hace Morin (1999) de conformar una Antropoética, surgiendo así otro cuestionamiento: ¿Qué es la Antropoética?

En este espacio consideramos manejar de manera sintética la noción de la antropoética, pues hacerlo de manera general será el motivo de otro espacio de reflexión, ya que al tratadista francés le ha llevado más de medio siglo de investigación, por ello nos concretaremos a decir que es la desvinculación de la idea del hombre jerárquicamente superior que han producido los humanismos del renacentismo, que sólo han llevado a la humanidad a los poderes extraordinarios que tiene de construirse así misma y su destino, pero lo hace caer en un desequilibrio, donde el hombre se incendia así mismo. (ROMERO, 2005, p. 10)

La Antropoética es concientizar al individuo de no ser un sujeto especial, jerárquicamente superior, si no un sujeto complejo, con un pensamiento complejo. Lo trata de concientizar que el conocimiento es una aventura que a cada instante corre el riesgo de confusión y simplificación, pues como lo señala 
Morin (1984, p. 320-330) el pensamiento es el arte de navegar entre confusión y abstracción, por ello la complejidad no es considerar al hombre como un ser biológico, ni tampoco como únicamente cultural, mucho menos como 50\% biológico y $50 \%$ cultural, sino como un ser totalmente biológico y totalmente antropológico, es decir metacultural, por ser cultural, espiritual, político, etc., donde cada uno de estos aspectos del hombre, se entrelazan formando el bucle que Morín (1999) nos explica gráficamente:

Individuo -- Sociedad -- Especie. Explicando que las flechas nos indican que estos términos se coproducen uno al otro sin jerarquizarlos. Por todo ello Morín (1984, p. 352-353) conceptualiza la Antropoética no como una ética únicamente humana, sino partiendo de que la idea de humanidad es totalmente biológica la cual tiene sus raíces en la unidad genético/cerebral de la especie "Homo" y totalmente metabiológica, incluso metasocial, puesto que rebasa el marco de las Sociedades, Estados e Imperios.

La complejidad de conceptualizar el término de la Antropoética, como ya lo dijimos es alta, ya que no sólo se constriñe a referir nuestros problemas humanos y sociales a un conocimiento de la naturaleza, particularmente biológico, sino que también se refiere a que este conocimiento de la naturaleza, particularmente biológico, lo llevemos a nuestras interacciones y determinaciones antroposociales. Por ende debemos entender que la Antropoética nos hace concientizar de que al mismo tiempo que somos producto de una evolución biológica, la noción de evolución biológica es producto de una evolución sociocultural, que no podemos ignorar el condicionamiento sociocultural del conocimiento científico, lo que hace que una teoría científica sea también una ideología de la sociedad. (MORÍN, 1984)

\section{LAS PROBLEMATICAS DE LA INVESTIGACIÓN EN HUMANIDADES}

A todas esas problemáticas, ya enunciadas en el punto anterior, propias de la investigación, se ha venido a agregar otras más con la denominada Reforma Integral de la Educación Media Superior (RIEMS), la cual las autoridades educativas enarbolan, como Miguel Székely, quien señala que la RIEMS forma parte de la urgente reforma educativa que necesita el país y que tiene por objetivo lograr que el bachillerato garantice la formación humanista de ciudadanos con un pensamiento crítico, autónomo, libre y reflexivo, para afrontar en mejores condiciones los desafios del siglo XXI. (AMADOR; GIL, 2009, p. 62-63) 
Sin embargo, en contraposición de la argumentación oficial se han pronunciado catedráticos de la UNAM, tratadistas y filósofos, así como diferentes agrupaciones académicas como el Observatorio Filosófico por La Defensa de La Filosofía, la Asociación de Filósofos Mexicanos, quienes aducen que es una reforma que obedece más a los lineamientos de la Organización para La Cooperación y el Desarrollo Económico (OCDE), los acuerdos de Bolonia y el Proyecto Tuning para la Unión Europea (AMADOR, GIL, ob.cit.), de que la reforma unificara todos los subsistemas a partir de la enseñanza por competencias y habilidades, su propósito es formar individuos para el mercado laboral, ya que el modelo de competencias está destinado a no formar seres humanos integrales, si no mano de obra para la producción.

Si la RIEMS es un instrumento para apoyar el plan nacional de desarrollo 2007-2012 cuyo objetivo es elevar la calidad educativa, entonces ¿Por qué decir que la RIEMS es una de las problemáticas de la investigación en humanidades? Ya hemos enunciado las argumentaciones en pro y en contra de la reforma, ahora para contestar al cuestionamiento, analizamos los acuerdos números 442 y 444 emitidos por la Secretaria de Educación Pública. El acuerdo 444 establece en sus considerandos que las competencias a que se refiere son parte del Marco Curricular Común que da sustento al Sistema Nacional de Bachillerato (SNB) eje en torno al cual se lleva a cabo la Reforma Integral de la Educación Media Superior. Hasta aquí todo marcha bien, pues la comunidad internacional se pronuncia por la Reforma Educativa ya que es una llamada de atención sobre la necesidad de convertir la acción educativa en una acción profunda y globalmente humanizadora (LUCINI, 1995, p. 5). Sin embargo, el artículo 7 del citado acuerdo enuncia de manera limitativa cuatro campos de disciplinas básicas de la siguiente forma:

\begin{tabular}{|c|c|}
\hline CAMPO DISCIPLINARIO & DISCIPLINAS \\
\hline Matemáticas & Matemáticas \\
\hline Ciencias Experimentales & Física, Química, Biología, y Ecología \\
\hline Ciencias Sociales & Historia, Sociología, Política, Economía, Administración \\
\hline Comunicación & $\begin{array}{c}\text { Lectura, Expresión Oral y Escrita, Literatura, } \\
\text { Lengua Extranjera e Informática }\end{array}$ \\
\hline
\end{tabular}

Como se puede observar de este cuadro de disciplinas básicas que establece el artículo 7 en comento, no aparecen las disciplinas humanísticas como la filo- 
sofía, la ética, la lógica y la estética, lo cual deja entrever el referido numeral que las incluye en las ciencias sociales, sin mencionarlas, otorgándoles así el carácter de transversales.

Tal carácter de transversales implica una problemática para la investigación, y en especial para la investigación en humanidades, ya que al no definir la transversalidad puede suceder lo que comenta F.G. Lucini $(1995$, p. 6) que las (os) profesoras (es) crean y formulen sus Proyectos Curriculares sobre los objetivos y los contenidos de las Áreas y al finalizarlos se preguntan ¿Cómo "metemos los transversales"? lo que el propio autor interpreta como un "parcheo" o una "chapuza" encaminada a cumplir con lo administrativo, sin observar lo educativo o formativo . Como bien lo afirma el filosofo José Luis Balcárcel (PONCE; PONCE, 2009, p. 64) se les denomina "Materias Transversales" sin saber exactamente que quieren decir con eso, pero si es cierto que equivale a que para los programas educativos de la SEP en bachillerato dejan de ser fundamentales, y por lo tanto pueden prescindir de esas materias en su enseñanza.

Otra problemática que encierra la RIEMS a la investigación, es la que cuestiona el tratadista Luis Villoro ¿Cuál es la base y la justificación de la filosofía?

La filosofia se presenta como la base de todas las ciencias y de todo conocimiento y se pregunta qué sentido tiene el mundo entero, para que todo esto. Se pregunta por el fin de todas las cosas, es una reflexión sobre el sentido y fin de todas las cosas. Pero seguimos cuestionando si la filosofía es relegada de las disciplinas básicas del SNB ¿Por qué es una problemática para la investigación? ¿Cuál es el vínculo que constriñe a la filosofía con la investigación? Como lo explica José Luis Balcárcel la enseñanza de la filosofía sigue siendo fundamental, además de ser un método para el mejor discernimiento o para conseguir ese discernimiento de problemas. Es decir, en cualquier disciplina e investigación no habría desarrollo, sin la enseñanza de la filosofía, ya que no podría haber planteamiento del problema. En palabras de R. Xirau (idem) sin la "filosofía" no hay posibilidad de conocer nada de lo demás.

Una tercera y última problemática que tocamos respecto de la RIEMS para la investigación es que estas denominadas "Materias Transversales" (Filosofía, Ética, Lógica y Estética) poseen ámbitos específicos que les corresponde investigar y enseñar (Balcárcel - idem -) tales ámbitos son los siguientes:

- Filosofía: es la base de todas las ciencias y por ende de todo conocimiento.

- Ética: es indispensable para estudiar los modos del comportamiento personal y social. 
- Lógica: es imprescindible para conocer y entender las estructuras o estructuración del pensamiento.

- Estética: ampara las formas de estudio del cómo se produce las relaciones de valoración con los objetos, sean estos artísticos, naturales o tecnológicos.

Por tanto estas Materias Transversales corresponden a formas del conocimiento que a través del desarrollo de la cultura demuestra su importancia para la investigación, generación y fundamentación del conocimiento. De tal modo que podríamos cuestionar ¿Cómo se podría generar el conocimiento o como podría desarrollarse la investigación sin la enseñanza de estas materias transversales humanísticas?

De esta demanda, reflexionamos que la RIEMS representa varias problemáticas para la investigación, ya que ¿Cómo podrá la investigación conformar la antropoética y afrontar la crisis del humanismo, si se prescinde de la enseñanza de estas materias de humanidades? ¿Cómo podrá la investigación lograr el reto de la conformación antropoética en los jóvenes estudiantes del bachillerato, para así solventar la crisis del humanismo?

Por las razones expuesta en los párrafos próximos-anteriores creemos que es prácticamente imposible que la investigación pueda llevarse a cabo y desarrollarse en cualesquier disciplina, sobre todo en las humanidades, para cumplir su cometido de generar conocimiento, para que sirva de enseñanza y formación de los estudiantes del SNB.

Consideramos que sin la enseñanza de las disciplinas de humanidades no se podrá formar los futuros investigadores, que en este momento son los estudiantes del bachillerato, debido a que estarán capacitados para la producción industrial o técnica, es decir, mano de obra altamente calificada para la producción de bienes materiales, capacitándoseles para pensar de manera dogmatica, más no como lo señala Juliana González (AMADOR; GIL, 2009, p. 61-63) de enseñar a los jóvenes a pensar de manera libre, formando ciudadanos consientes, autónomos y críticos, para la construcción del pensamiento, para desarrollar investigaciones, para construir la democracia, su libertad y por ende un mejor país y una mejor humanidad. Refuerza esta idea de la académica, lo afirmado por Luis Villoro (ídem) en el sentido de que esta formación conduce a la historia del pensamiento de la humanidad, pensamiento que lleva a cabo una acción colectiva, porque la filosofía no está separada de la acción de todo el mundo posible. No está separada la reflexión de la acción colectiva. 
Surge otro problema con la RIEMS en relación con la investigación, ya que sin la enseñanza de disciplinas humanísticas, uno se cuestionaría ¿Cómo podrá la investigación lograr la conformación de la antropoética? ¿Cómo afrontar la investigación, la crisis del humanismo, sin la enseñanza de las humanidades y sin una conformación antropoética?

Como ya lo hemos conceptualizado en el capitulo primero de este documento la antropoética es un término totalmente biológico, totalmente social, totalmente ético, totalmente humano (MORÍN, 1984) por lo que para su conformación en los futuros investigadores (actualmente estudiantes del SNB) se requiere una ardua labor de investigación en todas las disciplinas (no importando su clasificación en básicas o transversales), en los campos de la ciencia, la tecnología y las humanidades. El problema se vuelve aún más interesante desde el momento en que uno supone una relación indisoluble entre el mantenimiento de la estructura y el cambio de los constituyentes, y no nos abrimos a un problema clave, primordial, central, evidente, del ser viviente, problema que no sabemos si es ignorado u oculto por nuestras autoridades educativas, ya que la antigua física y la metafísica occidental (paradigma cartesiano) consideraban que todas las cosas vivientes son entidades cerradas y no los consideraban como sistemas abiertos que organizan su autonomía y su apertura. (MORIN, 2005, p. 44)

Dicho de otra manera, estas materias humanísticas son fundamentales en la investigación de la conformación antropoética de los jóvenes estudiantes del SNB, la cual consideramos el método más idóneo para afrontar la crisis del humanismo, pues como lo plantea la RIEMS se están coartando las posibilidades de crecimiento educativo, científico, tecnológico y de toda índole para millones de estudiantes actualmente son seis millones quinientos treinta y cuatro mil doscientos veinte estudiantes inscritos en el SNB, que oscilan entre los dieciséis y dieciocho años - (AMADOR; GIL) Así concluyendo, Juliana González (ídem) enfatiza que si en realidad se quiere una democracia robusta, verdadera y no simplemente un ejercicio de votar, se necesita formar a mexicanos con ética, con una formación que vaya más allá de la moral y que reflexione sobre los valores que se reciben en la casa o en las instituciones educativas, es decir, se requiere una conformación antropoética.

\section{RESULTADOS}

De todo lo aquí desarrollado se desprende que los acuerdos 442, principalmente el 444 de la SEP, no contemplan como disciplinas básicas a la Filosofía, Ética, Lógica y Estética. 
La implementación del SNB a través de la RIEMS no fue discutida plenamente por todos los actores y sectores de la educación, lo que repercute en la realización y logros de sus objetivos.

Se observó en este trabajo que la comunidad internacional se pronuncia por la Reforma Educativa, la cual señala como de vital importancia para fomentar la investigación.

La investigación se ve directamente afectada por la RIEMS, toda vez que al no contemplar como disciplinas básicas las materias de humanidades, se pone en riesgo el desarrollo integral de los estudiantes, con una conformación antropoética que les permita aplicarla y desarrollarla en sus investigaciones como futuros investigadores que lo serán.

\section{PROPUESTAS}

Creemos indudablemente en la urgente necesidad de la reforma educativa como la piedra angular que permitirá realizar los demás cambios y reformas que necesita no sólo el País, si no la Humanidad Planetaria. La RIEMS es una buena intención de reformar el Sistema Educativo, sin embargo, aún y cuando el subsecretario de Educación Media Superior de la SEP en turno (Miguel Székely) señala como imposible sustentar un dialogo directo de persona a persona, por tener una comunidad académica tan grande, proponemos la realización de mesas de trabajo, reuniones, asambleas, donde se trate de incluir a todos los actores de la educación y agrupaciones académicas en aras de enriquecer sin excluir, el debate educativo.

Así también proponemos que se abran más líneas de investigación en torno de la antropoética con el objetivo de demostrar su pertinencia en la conformación integral de los estudiantes de SNB, para formar antropoéticamente investigadores que puedan y sepan afrontar la crisis del humanismo.

\section{CONCLUSIONES}

La crisis del humanismo que aqueja los umbrales del siglo XXI, no constituye propiamente un problema sino consideramos que es un reto para la educación y por ende, para la investigación, especialmente la investigación en humanidades, por lo que la conformación antropoética de los alumnos del SNB surge como una estrategia de este escenario educativo.

Por lo tanto si la RIEMS no contempla como disciplinas básicas a las humanidades, zanja una problemática para la investigación, pues sin la enseñanza de las humanidades, difícilmente se podrá dar la conformación antropoética de 
los futuros investigadores, dejando al país sin estrategia viable para afrontar la crisis del humanismo.

\section{REFERENCIAS}

BOURDIEU, Pierre. Razones prácticas, sobre la teoría de la acción. Barcelona, España: Anagrama, 1999.

BOURDIEU, Pierre. Capital cultural, escuela y espacio social. México: Siglo XXI, 2000.

COMENIUS, Juan Amós. Didáctica magna. 15. ed. México: Porrúa, 2005.

DAMM ARNAL, Arturo. Libertad: esencia y existencia. 2. ed. México: Editora de Revistas S.A. de C.V., 1989.

CHOMSKY, Noam. El nuevo orden mundial (y el viejo). Barcelona: Critica, 2004.

GUARIGLIA, Osvaldo. Una ética para el siglo XXI. Ética y derechos humanos en un tiempo posmetafísico. México: Fondo de Cultura Económica, 2006.

ESTRADA, José Manuel. Libertad de enseñanza. En: LIBERTAD responsable y educación: nuevos desafíos para el docente. Buenos Aires: Montserrat, 1987.

FOUCAULT, Michel. Vigilar y castigar (nacimiento de la prisión). México: Siglo Veintiuno, 2004.

GIMENO SACRISTÁN, José; PÉREZ GÓMEZ, Angel I. Comprender y transformar la enseñanza. Madrid, España: Ediciones Morata, 1997.

GONZÁLEZ LUCINI, Fernando. La educación como tarea humanizadora, (de la teoría pedagógica a la práctica educativa). Madrid: Grupo Anaya, 2001.

GONZÁLEZ LUCINI, Fernando. Temas transversales y áreas curriculares. Madrid: Grupo Anaya, 1995. 
ILLICH, Ivan. 2006. La sociedad desescolarizada. México: Fondo de Cultura Económica, 2004.

LATAPI SARRE, Pablo. La moral regresa a la escuela. México: Centro de Estudios de la Universidad, Plaza y Valdez Editores, Universidad Nacional Autónoma de México, 2002.

LATAPI SARRE, Pablo. El debate sobre los valores en la escuela mexicana. México: Fondo de Cultura Económica, 2004.

LLANES TOVAR, Rafael. Como enseñar y transmitir los valores: guía para padres y maestros. México: Trillas, 2001.

MANNHEIM, Karl. Ideología y utopia. Introducción a la sociología del conocimiento. México: Fondo de Cultura Económica, 1987.

MORIN, Edgar. Introducción al pensamiento complejo. 8. ed. Barcelona: Gedisa, 2005.

MORIN, Edgar. El Método V. La humanidad de la humanidad: La identidad humana. [s. 1.] : Seuil, 2001.

MORIN, Edgar. Los siete saberes necesarios para una educación del futuro. UNESCO, 2000.

ROMERO, L. Necesario atender la crisis del humanismo. Gaceta UNAM, México, v. 3 n.860, Dic. 2005.

RUIZ, R., A; MARTÍNEZ, A.; CORRALES, V.; HERNÁNDEZ, V. B. Los estudios de posgrado en México: diagnóstico y perspectivas. UNESCOIESALC, IPN; UNAM. UAS. 2006. (En Prensa).

PONCE, R. Lo que piensan los filósofos... Processo, México, n. 1695, p. 64, abr. 2009.

PROCESO. México, n. 1707, 19 jul. 2009.

REVISTA INTERUNIVERSITARIA DE FORMACIÓN DE

PROFESORADO. Zaragoza: Universidad de Zaragoza, v. 18, n. 001. 